\title{
Strong-coupling phases of the $t-t^{\prime}$ Hubbard model
}

\author{
J. González \\ Instituto de Estructura de la Materia, CSIC, Serrano 123, 28006 Madrid, Spain \\ J. V. Alvarez \\ Escuela Politécnica Superior, Universidad Carlos III, Butarque 15, Leganés, 28913 Madrid, Spain
}

(Received 18 October 1996; revised manuscript received 29 January 1997)

\begin{abstract}
We study the strong-coupling regime of the $t$ - $t^{\prime}$ Hubbard model, filled up to the level of the van Hove singularities, by means of an exact diagonalization approach. We characterize the different phases of the model by the different sectors of the Hilbert space with given quantum numbers. By looking for the ground state of the system, we find essentially the competition between a state with incipient ferromagnetism and other mimicking a $d$-wave condensate, which has the lowest energy in a large region of the phase space.
\end{abstract} [S0163-1829(97)03925-8]

During recent years there have been increasingly accurate measures by angle-resolved photoemission spectroscopy of copper-oxide compounds, giving much insight into the phenomenology of these materials. ${ }^{1}$ Near the optimal doping for superconductivity the hole-doped compounds use to show extended van Hove singularities close to the Fermi level, which are located near the high-symmetry points $(\pi, 0),(0, \pi){ }^{1,2}$ On the other hand, in the carrier-free regime the materials show antiferromagnetic correlations, with a dispersion relation which has peaks at the points $( \pm \pi / 2, \pm \pi / 2){ }^{3}$ A most interesting problem is therefore to understand the drastic change that the Fermi surface may suffer by the influence of doping. 4

The framework that has been proposed to address such theoretical issues is that of the $t-t^{\prime}-U$ model $^{5}$ (or its strongcoupling version, the $t-t^{\prime}-J$ model $^{6}$ ), as it is generally believed that strong correlation effects have to be responsible for the electronic properties of the cuprates. Next-to-nearestneighbor hopping $t^{\prime}$ has to be introduced for a more accurate description of the dispersion relation in the insulating phase. ${ }^{7}$ The distinctive feature of the $t-t^{\prime}-U$ model is that the level of half-filling does not coincide with the level corresponding to the two van Hove singularities. Thus, it should be possible to establish a clearer separation between the effects of the antiferromagnetic correlations and those due to the appearance of the extended saddle points. There have been attempts to propose a purely electronic mechanism of superconductivity in systems with van Hove singularities close to the Fermi level. ${ }^{8-10}$ What is essential in those models is the existence of some enhanced channel favoring the exchange of singlet pairs. They represent an alternative to the picture earlier proposed in which the pairing interaction is supposed to arise from the short-range antiferromagnetic correlations. ${ }^{11}$

In the present paper we study the correlations which may dominate at the van Hove singularities, by performing the exact diagonalization of the $t-t^{\prime}$ Hubbard model in a $4 \times 4$ lattice (with periodic boundary conditions). The Hamiltonian of the model is

$$
H=-t \sum_{i, j, \sigma} c_{i \sigma}^{\dagger} c_{j \sigma}+t^{\prime} \sum_{i, j, \sigma} c_{i \sigma}^{\dagger} c_{j \sigma}+U \sum_{i} n_{i \uparrow} n_{i \downarrow}
$$

where $\sigma=\uparrow, \downarrow$, the first sum is over nearest neighbors $i, j$, the second sum over next-to-nearest neighbors, and $n_{i}$ is the electron number operator at site $i$. A typical contour map of the dispersion relation (for $t^{\prime}<0.5$ ) is shown in Fig. 1. We are especially interested in the situation in which the van Hove shell, comprising the four degenerate states at $(\pi, 0)$ and $(0, \pi)$, is half-filled. We will pursue the determination of the lowest-energy state in each of the sectors with different quantum numbers. By looking for the ground state of the model we will be able to study the interplay among the different phases of the system and, as long as we are relying on a quantity (the ground state energy) that probes the lattice as a whole, we may hope to predict properties with less influence from finite-size effects. This procedure is similar to that applied to the Hubbard model, ${ }^{12}$ where it appears to be safer than the evaluation of correlation functions on the small lattice scale. $^{13}$

Our starting point is the lattice in Fig. 1, filled with 12 particles for the 32 available one-particle states. For the noninteracting theory $(U=0)$, this means that the ground state is

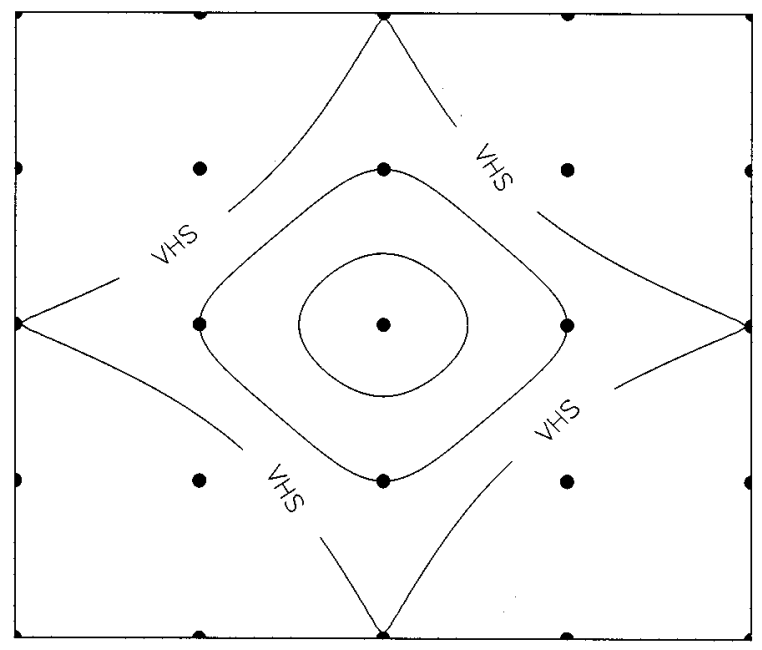

FIG. 1. Contour map of dispersion relation for the $t-t^{\prime}$ Hubbard model. The van Hove shell (VHS) is shown. 
sixfold degenerate. The six states are given by the different occupations of the van Hove points $A$ at $(\pi, 0)$ and $B$ at $(0, \pi)$. We may classify them according to the total momentum $\mathbf{P}$ and total spin $S$, ending up with a $S=1$ triplet at $\mathbf{P}=(\pi, \pi)$,

$$
\begin{gathered}
\left|T_{1}\right\rangle=c_{A \uparrow}^{\dagger} c_{B \uparrow}^{\dagger}|O\rangle, \\
\left|T_{0}\right\rangle=\frac{1}{\sqrt{2}}\left(c_{A \uparrow}^{\dagger} c_{B \downarrow}^{\dagger}+c_{A \downarrow}^{\dagger} c_{B \uparrow}^{\dagger}\right)|O\rangle, \\
\left|T_{-1}\right\rangle=c_{A \downarrow}^{\dagger} c_{B \downarrow}^{\dagger}|O\rangle,
\end{gathered}
$$

a spin singlet at $\mathbf{P}=(\pi, \pi)$,

$$
|P\rangle=\frac{1}{\sqrt{2}}\left(c_{A \uparrow}^{\dagger} c_{B \downarrow}^{\dagger}-c_{A \downarrow}^{\dagger} c_{B \uparrow}^{\dagger}\right)|O\rangle,
$$

and two spin singlets at $\mathbf{P}=(0,0)$

$$
\begin{aligned}
& |D\rangle=\frac{1}{\sqrt{2}}\left(c_{A \uparrow}^{\dagger} c_{A \downarrow}^{\dagger}-c_{B \uparrow}^{\dagger} c_{B \downarrow}^{\dagger}\right)|O\rangle, \\
& |S\rangle=\frac{1}{\sqrt{2}}\left(c_{A \uparrow}^{\dagger} c_{A \downarrow}^{\dagger}+c_{B \uparrow}^{\dagger} c_{B \downarrow}^{\dagger}\right)|O\rangle .
\end{aligned}
$$

The states $|D\rangle$ and $|S\rangle$ (as well as $\left|T_{0}\right\rangle$ and $|P\rangle$ ) differ in the quantum number associated with the transformation by exchange of the two components of the momentum, that is, one of the generators of the lattice symmetry group. The triplet state would be the precursor of a state with nonzero magnetization for systems with larger size, while $|P\rangle$ would correspond to a paramagnetic or antiferromagnetic state with no tendency to the uniform alignment of spins. On the other hand, the $|D\rangle$ state mimics the fluctuation of pairs between the two van Hove singularities, providing a simplified version of a $d$-wave condensate. In what follows we study how the degeneracy between these states is broken when the interaction is turned on, interpreting the corresponding ground state as an incipient signal of what should be the dominant correlation in the model.

By considering the interaction of the particles in the van Hove shell with the rest of closed shells in the Fermi sea (but without allowing yet for particle-hole screening processes) we see that the above ground-state degeneracy is partially lifted. To first order in $U$, the energy of each of the above multiplets is

$$
\begin{gathered}
E_{|T\rangle}=E_{\mathrm{kin}}+\frac{U}{N}\left(\frac{n_{F S}}{2}+2\right) \frac{n_{F S}}{2}+O\left(U^{2}\right), \\
\left.E_{|D\rangle}=E_{\mathrm{kin}}+\frac{U}{N}\left(\frac{n_{F S}}{2}+1\right)^{2}-1\right]+O\left(U^{2}\right), \\
\left.E_{|P\rangle}=E_{\mathrm{kin}}+\frac{U}{N}\left(\frac{n_{F S}}{2}+1\right)^{2}+1\right]+O\left(U^{2}\right), \\
E_{|S\rangle}=E_{\mathrm{kin}}+\frac{U}{N}\left[\left(\frac{n_{F S}}{2}+1\right)^{2}+1\right]+O\left(U^{2}\right),
\end{gathered}
$$

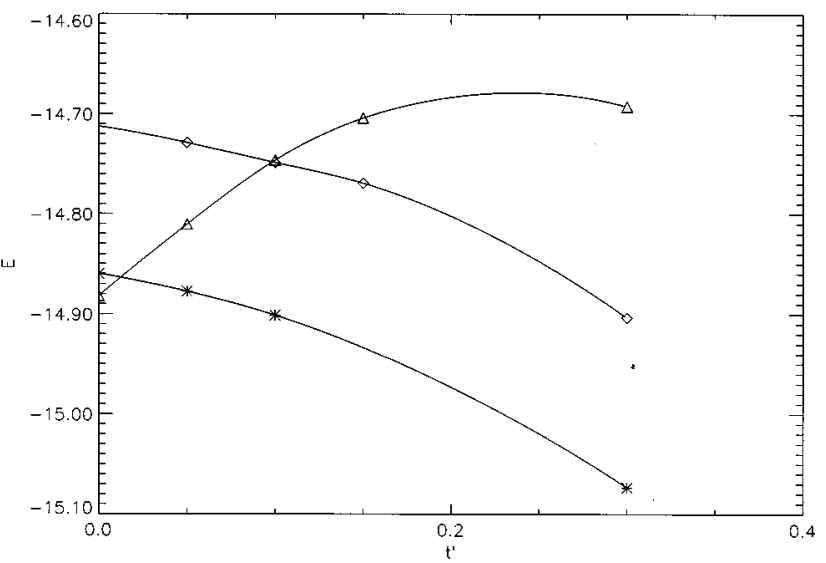

FIG. 2. Minimum value of energy in the sectors of $|D\rangle,|T\rangle$, and $|P\rangle$ states (asterisks, diamonds and triangles, respectively) in the region of small $t^{\prime}$ and $U=8 t$.

where $E_{\text {kin }}$ is the kinetic energy of the particles, $n_{F S}$ is the number of particles in closed shells, and $N$ is the number of lattice sites. We see that to this perturbative order the states $|T\rangle$ and $|D\rangle$ have both the lowest energy. If we had an extended saddle point, for instance, and the possibility of placing a number $m$ of particles near $A$ or $B$ in a more degenerate level, it is clear that aligning the spin of the $m$ particles would produce a state with an energy $\approx E_{\text {kin }}$ $+(U / N)\left(n_{F S} / 2+m\right) n_{F S} / 2+O\left(U^{2}\right)$, while any state made of a condensate of singlet pairs could hardly lower its energy from $\approx E_{\text {kin }}+(U / N)\left[\left(n_{F S}+m\right) / 2\right]^{2}+O\left(U^{2}\right)$. It seems therefore that, in the weak-coupling regime of a model with a high density of states close to the Fermi level, a state with a macroscopic amount of magnetization is energetically favored..$^{14}$ One has to bear in mind, however, that this argument works only for a weak-coupling constant. The influence of particle-hole processes and screening turns out to be crucial in the presence of the van Hove singularities. In our $4 \times 4$ lattice model one may check that, already at values of the coupling constant as small as $U \approx 2 t$, there is a value of $t^{\prime}$ below which the state corresponding to $|P\rangle$ dressed with particle-hole processes gets lower energy than that of $|T\rangle$. Our exact diagonalization study becomes relevant mainly in the strong-coupling regime, where there is no forecast of what may be the symmetry of the ground state.

As an illustration of the effects at large $U$ we have plotted in Fig. 2 the evolution of the minimum values of the energy in the different sectors with the respective quantum numbers of the $|T\rangle,|D\rangle$, and $|P\rangle$ states, in the region of small $t^{\prime}$ and $U=8 t$. From the computational point of view, we have reduced the Hilbert space in each case by using all the generators of the lattice symmetry group, ${ }^{15}$ and we have implemented a Davidson algorithm to obtain the lowest-energy state in each sector. The plot in Fig. 2 shows that above a certain value of $t^{\prime}$ the ground state is found in the sector with the symmetry of the $|D\rangle$ state and, in fact, it corresponds to the original $|D\rangle$ state in Eq. (4) dressed with a large amount of particle-hole processes from the Fermi sea of our model. However, in the region of very small $t^{\prime}(\leqslant 0.02 t)$, the lowest energy is reached in the sector of the $|P\rangle$ state. This is consistent with what we expect from the Hubbard model, that is 
what we recover in the limit $t^{\prime} \rightarrow 0$, although with a significant deviation from half-filling. The ground state that we find in that regime corresponds to what should be a state with paramagnetic or antiferromagnetic properties in larger lattices. It is worthwhile to remark that such a state becomes a highly excited state in our study for sufficiently large values of the next-to-nearest-neighbor hopping, being also overriden by the lowest-energy state with spin $S=1$ above some value of $t^{\prime}$. The two crossings of levels that we appreciate in Fig. 2 have a genuine quantum character, since we have already seen that at a very-weak-coupling constant the ground state has to become essentially either the triplet state in Eqs. (2) or the $|D\rangle$ state in Eq. (4).

We have to stress, though, that the results we obtain as a function of the $t^{\prime}$ parameter may serve only as a qualitative guide for what have to be the correct physical picture in larger lattices. The condition of sticking at the level of the van Hove singularities, for instance, implies in general that the filling level needs to be adjusted as the $t^{\prime}$ parameter is varied. The simplicity of our model, however, makes the particle number insensitive to changes in $t^{\prime}$, so that the van Hove shell is half-filled with a total of 12 particles for the 32 available one-particle states (corresponding to $\langle n\rangle=0.75$ ) up to $t^{\prime}=0.5 t$. We obtain the correct physical picture in the limit of the Hubbard model $t^{\prime} \rightarrow 0$, where the distribution of one-particle levels of the $4 \times 4$ lattice is quite even in the spectrum. It is less clear, though, the dependence that the ground state description may have on lattice size for large values of $t^{\prime}$ where the dispersion relation is significantly distorted, especially for values close to $t^{\prime}=0.5 t$ where the Fermi line through the van Hove points degenerates into a pair of straight lines. In this low-density regime one may not expect much influence from particle-hole processes and screening effects, while the degeneration of states at the bottom of the dispersion relation could favor the appearance of a ferromagnetic instability, ${ }^{16}$ leading to a modification in the larger lattices of the trend observed in Fig. 2 (see below).

Another issue is the fact that the Fermi line may also change with respect to the noninteracting form, depending on the value of $U$. A quantitative determination of the Fermi line is obviously unfeasible in the small lattice studied, but it makes sense otherwise to consider how the momentum distribution function deviates from the noninteracting case. We have taken for this purpose the ground state corresponding to the values $t^{\prime}=0.3 t$ and $U=10 t$, computing the values of $n(\mathbf{k})$ over the points available in the Brillouin zone. The distribution function is represented in Fig. 3. It becomes clear that the values of $n(\mathbf{k})$ for the unoccupied states at $U=0$ differ only slightly from zero, even in the present strong-coupling situation. Thus, although the momentum distribution function may suffer from significant finite-size effects, it seems consistent to consider that the van Hove shell is half-filled in our model with the above-mentioned number of particles. It turns out, on the other hand, that this is the relevant filling to study pairing effects at the van Hove singularities since adding or removing two more particles would result in a closed-shell configuration with a wellestablished ground state.

We have studied the behavior of the model at increasing values of $U$ and next-to-nearest-neighbor hopping $t^{\prime}=0.3 t$, which is adequate for making contact with the phenomenol-

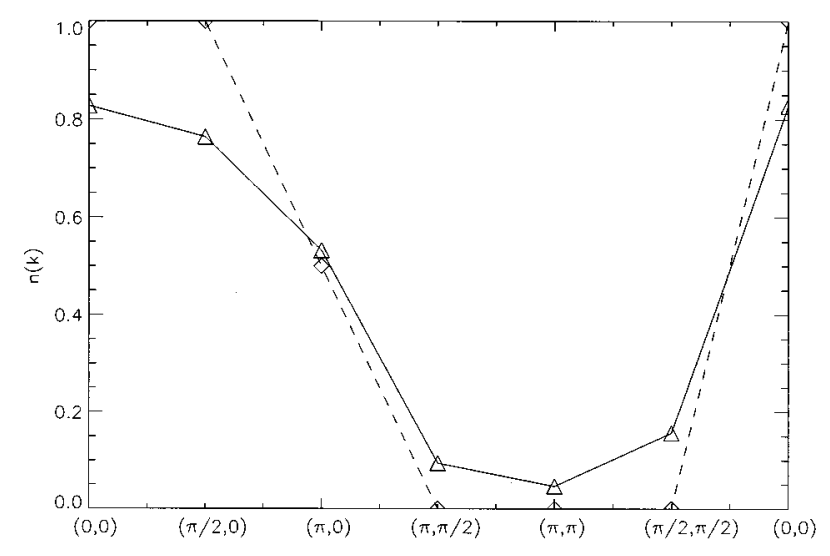

FIG. 3. Occupation number $n(\mathbf{k})$ for the ground state with $t^{\prime}=0.3 t, U=10 t$ (solid line). The values in the noninteracting case (dashed line) are also shown for comparison.

ogy of the cuprates. The sectors which may have candidates for the ground state of the system have again the quantum numbers of $|T\rangle,|D\rangle$, and $|P\rangle$, at least up to $U=12 t$. It can be checked that states with momentum different from $(0,0)$ or $(\pi, \pi)$ or spin different from 0 or 1 have much higher energy. Furthermore, any state with the quantum numbers of $|S\rangle$ is always an excited state of the system. In the mentioned range of the coupling constant, the ground state turns out to be the $|D\rangle$ state conveniently dressed by particle-hole processes from the closed shells of the Fermi sea. We have represented in Fig. 4 the difference in energy of such a state with the state of minimum energy in the sector of $|P\rangle$ and in Fig. 5 with respect to the corresponding lowest-energy state built from $|T\rangle$. It becomes clear that the state which could signal the appearance of antiferromagnetic order has no chance of being the ground state of the system, for the value of $t^{\prime}=0.3 t$ considered. Moreover, the results plotted in Fig. 5 show that the state which mimics the $d$-wave condensate has always lower energy than the triplet state up to the largest value $U=10 t$ considered. It is important to note that at the largest values of $U$ the energy difference between the two levels has a very slow decline, which makes it unlikely

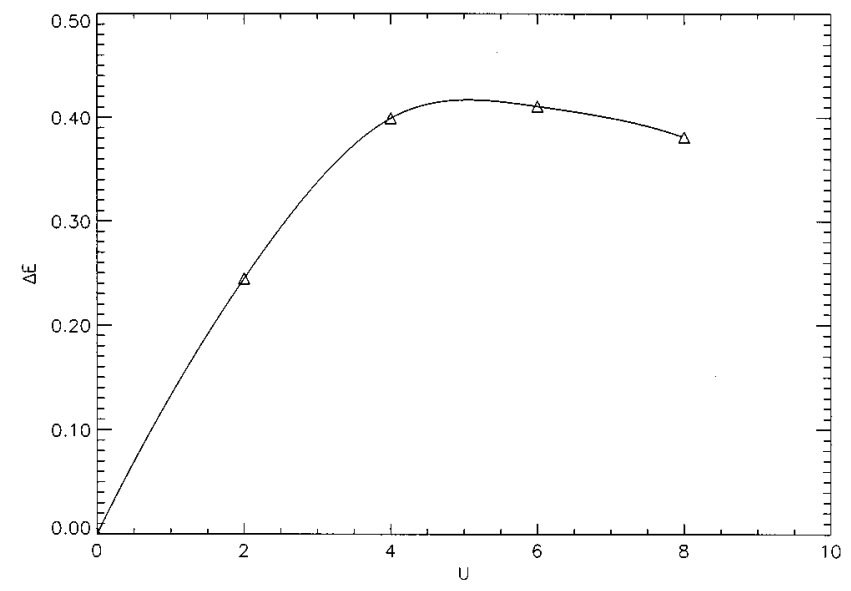

FIG. 4. Difference between minimum energies of the sectors containing $|P\rangle$ and $|D\rangle$ states $\left(t^{\prime}=0.3 t\right)$. 


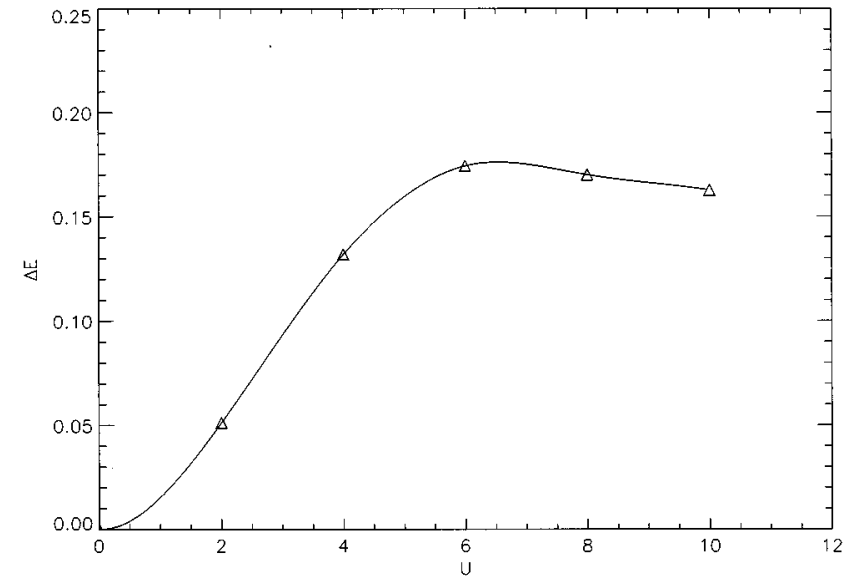

FIG. 5. Difference between minimum energies of the sectors containing $|T\rangle$ and $|D\rangle$ states $\left(t^{\prime}=0.3 t\right)$.

that the triplet state may become the ground state for a near value of $U$. The situation seems similar to the case of Nagaoka ferromagnetism, where this effect is found in the small $4 \times 4$ lattice for very large values of $U(>60 t)$, although the nature of the ferromagnetism may be quite different in the present model. The persistence of the $|D\rangle$ state as the ground state of the system over a large region of the parameter space provides good reason to believe that the corresponding $d$-wave condensate may continue playing that role in the larger lattices.

The possible crossing of levels between the singlet with $d$-wave symmetry and the $S=1$ state is also reminiscent of a phenomenon studied in small clusters of the hexagonal lattice. In those systems the regime in which the singlet prevails as the ground state has been linked to the effect of pair binding above half-filling. ${ }^{17}$ The hexagonal lattice at half-filling has strictly two Fermi points, which makes it likely that such a pair binding may rely on the resonance of electron pairs at the Fermi level. The important difference with respect to the model with the two van Hove singularities is that the density of states for the hexagonal lattice goes to zero at the Fermi points, which is just the opposite of the situation that we are facing. This may explain why the ground state with pairs fluctuating between the two van Hove points is more stable in this case, persisting over a much wider range of values of $U$ than in the clusters studied in Ref. 17.

The results that we have obtained have to be taken anyhow with the reserve inherent to the use of a small lattice in the exact diagonalization approach. As we have pointed out before, our analysis is likely to be relevant in the study of the strong-coupling regime, where the effects of screening due to particle-hole processes are difficult to assess by any other method. Moreover, our results apply directly to the zerotemperature regime of the system, while the use of other approaches like the quantum Monte Carlo method make it very difficult to perform extrapolations to the limit of vanishing temperature. This drawback seems to be overcome anyhow in the study of the $t-t^{\prime}$ Hubbard model of Ref. 16, where it is claimed that at $U=4 t$ and $t^{\prime}=0.47 t$ a signal of ferromagnetism is present in the model. For those values of the couplings our picture does not differ much qualitatively from what is shown in Figs. 4 and 5, but this is consistent with the fact that the signal found in Ref. 16 is so weak that requires lattices as large as $16 \times 16$ for the ferromagnetism to be observed. It has to be stressed that the value $t^{\prime}=0.47 t$ is very close to the point in which the Fermi sea degenerates into a pair of straight lines, making the analysis of the scattering processes of Ref. 16 in terms of ladder diagrams quite appropriate. Our approach, however, has to be relevant for a completely different regime, which arises when particle-hole processes induce strong screening effects in the model. ${ }^{18}$

Of course, in an ideal analytical framework one should be able to deal simultaneously with the description of screening processes and scattering in the particle-particle channel. One way to accomplish this would be to adopt a renormalization group approach, taking first into account the scaling of the different channels and studying then the behavior of the response functions. The two channels that are not irrelevant, apart from the forward scattering channel, ${ }^{19}$ are the scattering $V$ of pairs about the same van Hove singularity and the scattering $\widetilde{V}$ of pairs from one singularity to the other. The regime in which the intrasingularity screening dominates making $V<\widetilde{V}$ (which happens above certain value of $t^{\prime}$ ) seems to lead to an instability favoring the condensation of pairs with opposite amplitude in the two van Hove singularities. ${ }^{9}$ This is nothing but a new version of the KohnLuttinger mechanism, ${ }^{20}$ in which the anisotropy of the screening effects arises in a natural way. The superconducting instability may therefore be competing with the abovementioned ferromagnetic instability, at least for values of $t^{\prime}$ not very close to $0.5 t$. It would be worthwhile to develop some computational scheme with which to perform an extrapolation of our results to larger lattice sizes, in order to see whether the evidence found for $d$-wave superconductivity survives in the strong-coupling regime.

We want to thank F. Guinea for his collaboration at the early stages of this work. Useful discussions with R. Hlubina and S. Sorella are also acknowledged.
${ }^{1}$ Z.-X. Shen et al., Science 267, 343 (1995), and references therein.

${ }^{2}$ K. Gofron et al., Phys. Rev. Lett. 73, 3302 (1994).

${ }^{3}$ B. O. Wells et al., Phys. Rev. Lett. 74, 964 (1995).

${ }^{4}$ X.-G. Wen and P. A. Lee, Phys. Rev. Lett.76, 503 (1996); R. Hayn, A. F. Barabanov, and J. Schulenburg, Z. Phys. B 102, 359 (1997).

${ }^{5}$ P. Bénard, L. Chen, and A. M. Tremblay, Phys. Rev. B 47, 15
217 (1993); Q. Si, T. Zha, K. Levin, and J. P. Lu, ibid. 47, 9055 (1993); D. Duffy and A. Moreo, ibid. 52, 15607 (1995); A. F. Veilleux, A.-M. Daré, L. Chen, Y. M. Vilk, and A.-M. S. Tremblay, ibid. 52, 16255 (1995).

${ }^{6}$ T. Tanamoto, K. Kuboki, and H. Fukuyama, J. Phys. Soc. Jpn. 60, 3072 (1991); E. Gagliano, S. Bacci, and E. Dagotto, Phys. Rev. B 42, 6222 (1990); T. Tokyama and S. Maekawa, ibid. 49, 3596 (1994); M. Lavagna and G. Stemmann, ibid. 49, 4235 (1994); G. 
Stemmann, C. Pépin, and M. Lavagna, ibid. 50, 4075 (1994); R. J. Gooding, K. J. E. Vos, and P. W. Leung, ibid. 49, 4119 (1994); R. J. Gooding, K. J. E. Vos, and P. W. Leung, ibid. 50, 12866 (1994).

${ }^{7}$ A. Nazarenko, K. J. E. Vos, S. Haas, E. Dagotto, and R. J. Gooding, Phys. Rev. B 51, 8676 (1995).

${ }^{8}$ D. M. Newns, H. R. Krishnamurthy, P. C. Pattnaik, C. C. Tsuei, and C. L. Kane, Phys. Rev. Lett. 69, 1264 (1992).

${ }^{9}$ J. González, F. Guinea, and M. A. H. Vozmediano, Europhys. Lett. 34, 711 (1996).

${ }^{10}$ D. Z. Liu and K. Levin (unpublished).

${ }^{11}$ N. E. Bickers, D. J. Scalapino, and S. R. White, Phys. Rev. Lett. 62, 961 (1989); E. Dagotto, J. Riera, and A. P. Young, Phys. Rev. B 42, 2347 (1990); P. Monthoux and D. Pines, Phys. Rev. Lett. 69, 961 (1992); E. Dagotto and J. Riera, ibid. 70, 682 (1993); E. Dagotto, A. Nazarenko, and A. Moreo, ibid. 74, 310 (1995)

${ }^{12}$ A. Parola, S. Sorella, M. Parrinello, and E. Tosatti, Phys. Rev. B 43, 6190 (1991).

${ }^{13}$ See, for instance, the discussion in G. Fano, F. Ortolani, and A. Parola, Phys. Rev. B 42, 6877 (1990).

${ }^{14}$ This is reminiscent of the flat band ferromagnetism studied by A.
Mielke, Phys. Lett. A 174, 443 (1993); H. Tasaki, Phys. Rev. Lett. 69, 1608 (1992); A. Mielke and H. Tasaki, Commun. Math. Phys. 158, 341 (1993); H. Tasaki, Phys. Rev. Lett. 75, 4678 (1995). See also P. Pieri, S. Daul, D. Baeriswyl, M. Dzierzawa, and P. Fazekas, Phys. Rev. B 54, 9250 (1996).

${ }^{15}$ G. Fano, F. Ortolani, and A. Parola, Phys. Rev. B 46, 1048 (1992).

${ }^{16}$ R. Hlubina, S. Sorella, and F. Guinea, Phys. Rev. Lett. 78, 1343 (1997).

${ }^{17}$ S. R. White, S. Chakravarty, M. P. Gelfand, and S. A. Kivelson, Phys. Rev. B 45, 5062 (1992).

${ }^{18}$ After completion of this work we have been aware of quantum Monte Carlo computations for the $t-t^{\prime}$ model with values of $t^{\prime}$ in the range considered in this paper and $U$ up to $3 t$, by $\mathrm{T}$. Husslein, I. Morgenstern, D. M. Newns, P. C. Pattnaik, J. M. Singer, and H. G. Matuttis, Phys. Rev. B 54, 16179 (1996). For filling levels where the Fermi energy lies close to the van Hove singularity, a pairing tendency in the $d$ symmetry channel is found by these authors.

${ }^{19}$ R. Shankar, Rev. Mod. Phys. 66, 129 (1994).

${ }^{20}$ W. Kohn and J. M. Luttinger, Phys. Rev. Lett. 15, 524 (1965). 